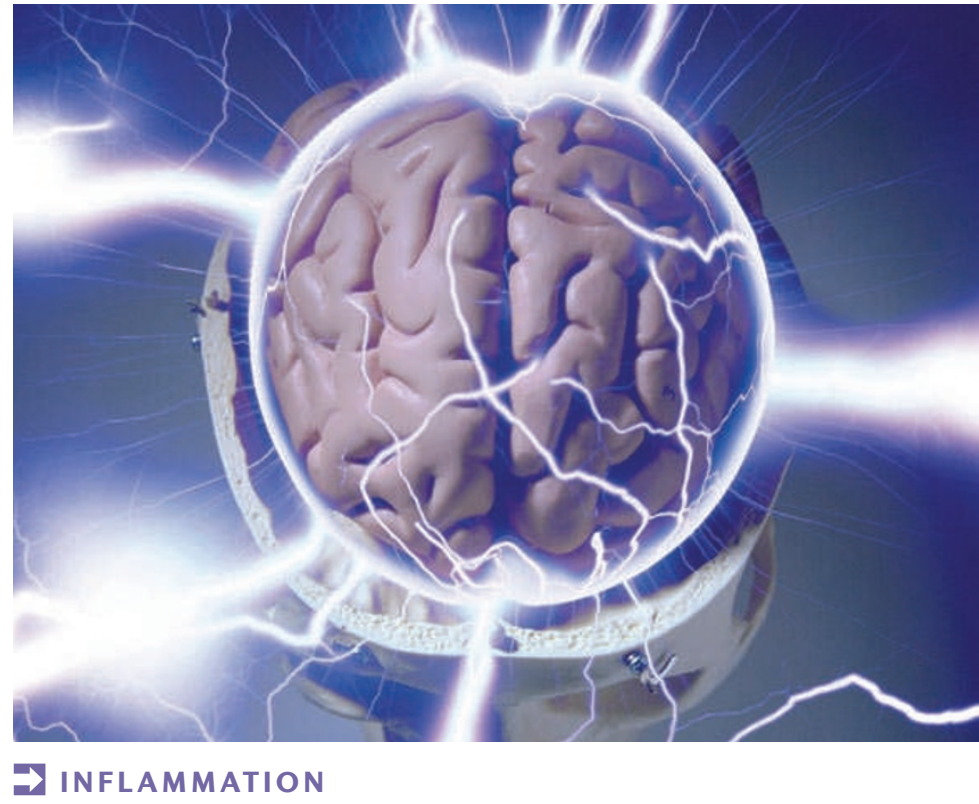

\section{An innate signal for CNS disease}

Multiple sclerosis and the animal model experimental autoimmune encephalomyelitis (EAE) are thought to develop in genetically predisposed individuals after an environmental trigger activates myelin-specific $\mathrm{T}$ cells in the central nervous system (CNS). A recent study published in Immunity suggests that peptidoglycan (PGN), a component of bacterial cell walls, activates antigenpresenting cells (APCs) in the CNS to provide this trigger.

PGN is recognized by the pattern recognition receptors nucleotidebinding oligomerization domain protein 1 (NOD1), NOD2 and Toll-like receptor 2 (TLR2), and it initiates a pro-inflammatory signalling cascade downstream of the NOD proteins that is mediated by receptor-interacting protein 2 (RIP2; encoded by Ripk2). To investigate the role of PGN in neuroinflammation, the authors analysed the development of EAE in mice lacking the PGN sensors or the downstream signalling adaptor protein. The incidence of disease after EAE induction was similar in wild-type and gene-deficient

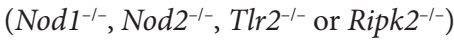
mice. However, the disease severity was reduced in all of the mutant mice, with Ripk $2^{-/-}$mice developing the mildest disease.
Experiments to determine the cause of this protection from disease progression in the gene-deficient mice revealed that it was not due to a $\mathrm{T}$ cell-intrinsic defect, as no differences in the development, proliferation, differentiation or CNS infiltration of myelin-specific T cells were observed between wild-type and mutant mice shortly after disease induction. However, 17 days after disease induction, fewer $\mathrm{CD} 4^{+} \mathrm{T}$ cells were observed in the CNS of $\mathrm{Nod}^{-1-}$, Nod2 $2^{-/-}$and Ripk2 $2^{-/-}$mice than in the CNS of wild-type mice.

In parallel with this decrease in $\mathrm{T}$ cell numbers, the mutant mice had fewer CD11 $c^{+}$dendritic cells (DCs) in the CNS, with the most marked reduction occurring in Ripk $2^{-/-}$mice. Ripk2 $2^{-l-}$ DCs did not show defective migration but instead seemed to be less activated (as determined by levels of MHC class II expression) than wild-type DCs. Moreover, Ripk2 ${ }^{-/-}$DCs had an impaired ability to drive naive $\mathrm{T}$ cell differentiation into T helper 17 cells in vitro.

Finally, evidence from bone marrow chimeras and adoptive transfer experiments confirmed that EAE progression depends on RIP2 activation in peripheral APCs, which migrate to the CNS and promote the reactivation and expansion of effector T cells.

\section{Lucy Bird}

ORIGINAL RESEARCH PAPER Shaw, P. J. et al. Signaling via the RIP2 adaptor protein in central nervous system-infiltrating dendritic cells promotes inflammation and autoimmunity. Immunity 34, 75-84 (2011) 